Kragujevac Journal of Mathematics

Volume 38(1) (2014), Pages 125-146.

\title{
SOME NEW HERMITE-HADAMARD TYPE INEQUALITIES FOR FUNCTIONS WHOSE HIGHER ORDER PARTIAL DERIVATIVES ARE CO-ORDINATED $s$-CONVEX
}

\author{
MUHAMMAD AMER LATIF
}

\begin{abstract}
In this paper we point out some inequalities of Hermite-Hadamard type for double integrals of functions whose partial derivatives of higher order are co-ordinated $s$-convex in the second sense. Our established results generalize the Hermite-Hadamard type inequalities established for co-ordinated $s$-convex functions and refine those results established for differentiable functions whose partial derivatives of higher order are co-ordinated convex proved in recent literature.
\end{abstract}

\section{INTRODUCTION}

A function $f: I \rightarrow \mathbb{R}, \emptyset \neq I \subseteq \mathbb{R}$, is said to be convex on $I$ if the inequality

$$
f(\lambda x+(1-\lambda) y) \leq \lambda f(x)+(1-\lambda) f(y),
$$

holds for all $x, y \in I$ and $\lambda \in[0,1]$. The inequality (1.1) holds in reverse direction if $f$ is concave.

The most famous inequality concerning the class of convex functions, is the HermiteHadamard's inequality.

This double inequality is stated as

$$
f\left(\frac{a+b}{2}\right) \leq \frac{1}{b-a} \int_{a}^{b} f(x) d x \leq \frac{f(a)+f(b)}{2}
$$

where $f: I \rightarrow \mathbb{R}, \emptyset \neq I \subseteq \mathbb{R}$ a convex function, $a, b \in I$ with $a<b$. The inequalities in (1.2) are in reversed order if $f$ a concave function.

The inequalities (1.2) have become an important cornerstone in mathematical analysis and optimization and many uses of these inequalities have been discovered in a

\footnotetext{
Key words and phrases. Convex function, Hermite-Hadamard type inequality, Co-ordinated convex function, $s$-convex function, Power-mean integral inequality.

2010 Mathematics Subject Classification. 26A33, 26A51, 26D07, 26D10, 26D15.

Received: June 10, 2013

Revised: June 20, 2014.
} 
variety of settings. Moreover, many inequalities of special means can be obtained for a particular choice of the function $f$. Due to the rich geometrical significance of HermiteHadamard's inequality (1.2), there is growing literature providing its new proofs, extensions, refinements and generalizations, see for example [8, 14, 19, 29, 32, 33] and the references therein.

In the paper [15], Hudzik and Maligranda considered, among others, the class of functions which are $s$-convex in the second sense. This class is defined follows.

A function $f:[0, \infty) \rightarrow \mathbb{R}$ is said to be $s$-convex in the second sense if

$$
f(\lambda x+(1-\lambda) y) \leq \lambda^{s} f(x)+(1-\lambda)^{s} f(y)
$$

holds for all $x, y \in[0, \infty), \lambda \in[0,1]$ and for some fixed $s \in(0,1]$.

It can be easily seen that for $s=1, s$-convexity reduces to ordinary convexity of functions defined on $[0, \infty)$.

In [9], Dragomir and Fitzpatrick proved a variant of Hadamard's inequality which holds for $s$-convex functions in the second sense.

Theorem 1.1. [9] Suppose that $f:[0, \infty) \rightarrow[0, \infty)$ is an s-convex function in the second sense, where $s \in(0,1)$ and $a, b \in[0, \infty), a<b$. If $f \in L^{1}[a, b]$, then the following inequalities hold

$$
2^{s-1} f\left(\frac{a+b}{2}\right) \leq \frac{1}{b-a} \int_{a}^{b} f(x) d x \leq \frac{f(a)+f(b)}{s+1} .
$$

The constant $k=\frac{1}{s+1}$ is the best possible in the second inequality in (1.3).

For more about properties and Hermite-Hadamard type inequalities of $s$-convex functions in the second sense we refer the interested readers to $[7,9,12,15,20]$.

Let us consider now a bidimensional interval $\Delta=:[a, b] \times[c, d]$ in $\mathbb{R}^{2}$ with $a<b$ and $c<d$. A mapping $f: \Delta \rightarrow \mathbb{R}$ is said to be convex on $\Delta$ if the inequality

$$
f(\lambda x+(1-\lambda) z, \lambda y+(1-\lambda) w) \leq \lambda f(x, y)+(1-\lambda) f(z, w)
$$

holds for all $(x, y),(z, w) \in \Delta$ and $\lambda \in[0,1]$.

A modification for convex functions on $\Delta$, known as co-ordinated convex functions, was introduced by S. S. Dragomir [10] as follows.

A function $f: \Delta \rightarrow \mathbb{R}$ is said to be convex on the co-ordinates on $\Delta$ if the partial mappings $f_{y}:[a, b] \rightarrow \mathbb{R}, f_{y}(u)=f(u, y)$ and $f_{x}:[c, d] \rightarrow \mathbb{R}, f_{x}(v)=f(x, v)$ are convex where defined for all $x \in[a, b], y \in[c, d]$.

A formal definition for co-ordinated convex functions may be stated as follow.

Definition 1.1. [21] A function $f: \Delta \rightarrow \mathbb{R}$ is said to be convex on the co-ordinates on $\Delta$ if the following inequality holds for all $t, r \in[0,1]$ and $(x, u),(y, w) \in \Delta$

$$
\begin{aligned}
f(t x+(1-t) y, r u+(1-r) w) \leq & t r f(x, u)+t(1-r) f(x, w)+r(1-t) f(y, u) \\
& +(1-t)(1-r) f(y, w) .
\end{aligned}
$$


Clearly, every convex mapping $f: \Delta \rightarrow \mathbb{R}$ is convex on the co-ordinates but converse may not be true [10].

The following Hermite-Hadamard type inequalities for co-ordinated convex functions on the rectangle from the plane $\mathbb{R}^{2}$ were established in [10].

Theorem 1.2. [10] Suppose that $f: \Delta \rightarrow \mathbb{R}$ is co-ordinated convex on $\Delta$, then

$$
\begin{aligned}
f\left(\frac{a+b}{2}, \frac{c+d}{2}\right) \leq & \frac{1}{2}\left[\frac{1}{b-a} \int_{a}^{b} f\left(x, \frac{c+d}{2}\right) d x+\frac{1}{d-c} \int_{c}^{d} f\left(\frac{a+b}{2}, y\right) d y\right] \\
\leq & \frac{1}{(b-a)(d-c)} \int_{a}^{b} \int_{c}^{d} f(x, y) d y d x \\
\leq & \frac{1}{4}\left[\frac{1}{b-a} \int_{a}^{b}[f(x, c)+f(x, d)] d x\right. \\
& \left.+\frac{1}{d-c} \int_{c}^{d}[f(a, y)+f(b, y)] d y\right] \\
\leq & \frac{f(a, c)+f(a, d)+f(b, c)+f(b, d)}{4} .
\end{aligned}
$$

The above inequalities are sharp.

The concept of $s$-convex functions on the co-ordinates in the second sense was introduced by Alomari and Darus in [3] as a generalization of the usual co-ordinated convexity.

Definition 1.2. [3] Consider the bidimensional interval $\Delta=[a, b] \times[c, d]$ in $[0, \infty)^{2}$ with $a<b$ and $c<d$. The mapping $f: \Delta \rightarrow \mathbb{R}$ is $s$-convex in the second sense on $\Delta$ if $f(\lambda x+(1-\lambda) z, \lambda y+(1-\lambda) w) \leq \lambda^{s} f(x, y)+(1-\lambda)^{s} f(z, w)$, holds for all $(x, y),(z, w) \in \Delta, \lambda \in[0,1]$ with some fixed $s \in(0,1]$.

A function $f: \Delta \subseteq[0, \infty)^{2} \rightarrow \mathbb{R}$ is called $s$-convex in the second sense on the co-ordinates on $\Delta$ if the partial mappings $f_{y}:[a, b] \rightarrow \mathbb{R}, f_{y}(u)=f(u, y)$ and $f_{x}$ : $[c, d] \rightarrow \mathbb{R}, f_{x}(v)=f(x, v)$, are $s$-convex in the second sense for all $y \in[c, d], x \in[a, b]$ and $s \in(0,1]$, i.e., the partial mappings $f_{y}$ and $f_{x}$ are $s$-convex in the second sense with some fixed $s \in(0,1]$.

A formal definition of co-ordinated $s$-convex function in second sense may be stated as follows.

Definition 1.3. A function $f: \Delta \subseteq[0, \infty)^{2} \rightarrow \mathbb{R}$ is called $s$-convex in the second sense on the co-ordinates on $\Delta$ if

$$
\begin{aligned}
f(t x+(1-t) y, r u+(1-r) w) \leq & t^{s} r^{s} f(x, u)+t^{s}(1-r)^{s} f(x, w) \\
& +r^{s}(1-t)^{s} f(y, u)+(1-t)^{s}(1-r)^{s} f(y, w)
\end{aligned}
$$

holds for all $t, r \in[0,1]$ and $(x, u),(y, u),(x, w),(y, w) \in \Delta$, for some fixed $s \in(0,1]$. The mapping $f$ is concave on the co-ordinates on $\Delta$ if the inequality (1.5) holds in reversed direction for all $t, r \in[0,1]$ and $(x, y),(u, w) \in \Delta$ with some fixed $s \in(0,1]$. 
Furthermore, Alomari and Darus [5] introduced a new class of $s$-convex functions on the co-ordinates on the rectangle from the plane as follows.

Definition 1.4. [5] Consider the bidimensional interval $\Delta=:[a, b] \times[c, d]$ in $[0, \infty)^{2}$ with $a<b$ and $c<d$. The mapping $f: \Delta \rightarrow \mathbb{R}$ is $s$-convex in the second sense on $\Delta$ if there exist $s_{1}, s_{2} \in(0,1]$ with $s=\frac{s_{1}+s_{2}}{2}$ such that

$$
f(\lambda x+(1-\lambda) z, \lambda y+(1-\lambda) w) \leq \lambda^{s_{1}} f(x, y)+(1-\lambda)^{s_{2}} f(z, w)
$$

holds for all $(x, y),(z, w) \in \Delta, \lambda \in[0,1]$. This class of functions is denoted by $M W O_{s_{1}, s_{2}}^{2}$.

A function $f: \Delta \subseteq[0, \infty)^{2} \rightarrow \mathbb{R}$ is called $s$-convex in the second sense on the co-ordinates on $\Delta$ if the partial mappings $f_{y}:[a, b] \rightarrow \mathbb{R}, f_{y}(u)=f(u, y)$ and $f_{x}$ : $[c, d] \rightarrow \mathbb{R}, f_{x}(v)=f(x, v)$, are $s_{1}$-convex and $s_{2}$-convex in the second sense for all $y \in[c, d], x \in[a, b]$ and $s_{1}, s_{2} \in(0,1]$ with $s=\frac{s_{1}+s_{2}}{2}$, respectively, i.e., the partial mappings $f_{y}$ and $f_{x}$ are $s_{1}$-convex and $s_{2}$-convex in the second sense, $s_{1}, s_{2} \in(0,1]$ with $s=\frac{s_{1}+s_{2}}{2}$.

The definition 1.3 can be generalized as follows.

Definition 1.5. A function $f: \Delta=:[a, b] \times[c, d] \subseteq[0, \infty)^{2} \rightarrow \mathbb{R}$ is called $s$-convex in the second sense on the co-ordinates on $\Delta$ if

$$
f(t x+(1-t) y, r u+(1-r) w) \leq t^{s_{1}} r^{s_{2}} f(x, u)+t^{s_{1}}(1-r)^{s_{2}} f(x, w)
$$

$$
+r^{s_{2}}(1-t)^{s_{1}} f(y, u)+(1-t)^{s_{1}}(1-r)^{s_{2}} f(y, w)
$$

holds for all $t, r \in[0,1]$ and $(x, u),(y, u),(x, w),(y, w) \in \Delta, s_{1}, s_{2} \in(0,1]$ with $s=\frac{s_{1}+s_{2}}{2}$. The mapping $f$ is concave on the co-ordinates on $\Delta$ if the inequality (1.6) holds in reversed direction for all $t, r \in[0,1]$ and $(x, y),(u, w) \in \Delta, s_{1}, s_{2} \in(0,1]$ with $s=\frac{s_{1}+s_{2}}{2}$.

In [5], Alomari et al. also proved a variant of inequalities given above by (1.4) for $s$-convex functions in the second sense on the co-ordinates on a rectangle from the plane $\mathbb{R}^{2}$.

Theorem 1.3. [5] Suppose $f: \Delta \subseteq[0, \infty)^{2} \rightarrow[0, \infty)$ is s-convex function in the second sense on the co-ordinates on $\Delta$. Then one has the inequalities

$$
\begin{aligned}
\frac{4^{s_{1}-1}+4^{s_{2}-1}}{2} f\left(\frac{a+b}{2}, \frac{c+d}{2}\right) \leq & \frac{2^{s_{1}-2}}{b-a} \int_{a}^{b} f\left(x, \frac{c+d}{2}\right) d x \\
& +\frac{2^{s_{2}-2}}{d-c} \int_{c}^{d} f\left(\frac{a+b}{2}, y\right) d y \\
& \leq \frac{1}{(b-a)(d-c)} \int_{a}^{b} \int_{c}^{d} f(x, y) d y d x
\end{aligned}
$$




$$
\begin{aligned}
& \leq \frac{1}{2\left(s_{1}+1\right)}\left(\frac{1}{b-a} \int_{a}^{b}[f(x, c)+f(x, d)] d x+\frac{1}{d-c} \int_{c}^{d}[f(a, y)+f(b, y)] d y\right) \\
& \leq \frac{1}{2}\left(\frac{1}{\left(s_{1}+1\right)^{2}}+\frac{1}{\left(s_{2}+1\right)^{2}}\right)[f(a, c)+f(b, c)+f(a, d)+f(b, d)] .
\end{aligned}
$$

In recent years, many authors have proved several inequalities for co-ordinated convex functions. These studies include, among others, the works in $[1,3,4,5,6]$, [10], [13], [21]-[24], [25]-[28] and [31]. Alomari et al. [1, 3, 4, 5, 6], proved several Hermite-Hadamard type inequalities for co-ordinated $s$-convex functions and coordinated log-convex functions. Dragomir [10], proved the Hermite-Hadamard type inequalities for co-ordinated convex functions. Hwang et. al [13], also proved some Hermite-Hadamard type inequalities for co-ordinated convex function of two variables by considering some mappings directly associated to the Hermite-Hadamard type inequality for co-ordinated convex mappings of two variables. Latif et. al [12]-[14], proved some inequalities of Hermite-Hadamard type for differentiable co-ordinated convex functions, differentiable functions whose higher order partial derivatives are coordinated convex, product of two co-ordinated convex mappings and for co-ordinated $h$-convex mappings. Özdemir et. al [25]-[28], proved Hadamard's type inequalities for co-ordinated convex functions, co-ordinated $s$-convex functions and co-ordinated $m$-convex and $(\alpha, m)$-convex functions.

The main aim of this paper is to establish some new Hermite-Hadamard type inequalities for differentiable functions whose partial derivatives of higher order are co-ordinated $s$-convex in the second sense on the rectangle from the plane $\mathbb{R}^{2}$ which generalize the Hermite-Hadamard type inequalities proved for co-ordinated $s$-convex functions in the second sense and refine those results established for differentiable functions whose partial derivatives of higher order are co-ordinated convex on the rectangle from the plane $\mathbb{R}^{2}$ (see [24]).

\section{Main Results}

In this section we establish new Hermite-Hadamard type inequalities for double integrals of functions whose partial derivatives of higher order are co-ordinated $s$ convex in the second sense.

To make the presentation easier and compact to understand, we make some symbolic representations as follows

$$
\begin{aligned}
A^{\prime}= & \frac{1}{2}\left[\frac{1}{b-a} \int_{a}^{b}[f(x, c)+f(x, d)] d x+\frac{1}{d-c} \int_{c}^{d}[f(a, y)+f(b, y)] d y\right] \\
& +\frac{1}{2} \sum_{l=2}^{m-1} \frac{(l-1)(d-c)^{l}}{2(l+1) !}\left[\frac{\partial^{l} f(a, c)}{\partial y^{l}}+\frac{\partial^{l} f(b, c)}{\partial y^{l}}\right] \\
& +\frac{1}{2} \sum_{k=2}^{n-1} \frac{(k-1)(b-a)^{k}}{2(k+1) !}\left[\frac{\partial^{k} f(a, c)}{\partial x^{k}}+\frac{\partial^{k} f(a, d)}{\partial x^{k}}\right]
\end{aligned}
$$




$$
\begin{aligned}
& -\frac{1}{b-a} \sum_{l=2}^{m-1} \frac{(l-1)(d-c)^{l}}{2(l+1) !} \int_{a}^{b} \frac{\partial^{l} f(x, c)}{\partial y^{l}} d x \\
& -\frac{1}{d-c} \sum_{k=2}^{n-1} \frac{(k-1)(b-a)^{k}}{2(k+1) !} \int_{c}^{d} \frac{\partial^{k} f(a, y)}{\partial x^{k}} d y \\
& -\sum_{k=2}^{n-1} \sum_{l=2}^{m-1} \frac{(k-1)(l-1)(b-a)^{k}(d-c)^{l}}{4(k+1) !(l+1) !} \frac{\partial^{k+l} f(a, c)}{\partial x^{k} y^{l}}
\end{aligned}
$$

and

$$
\begin{aligned}
& B_{(n, m)}=\left|\frac{\partial^{n+m} f(a, c)}{\partial t^{n} \partial r^{m}}\right|, \quad C_{(n, m)}=\left|\frac{\partial^{n+m} f(a, d)}{\partial t^{n} \partial r^{m}}\right|, \quad D_{(n, m)}=\left|\frac{\partial^{n+m} f(b, c)}{\partial t^{n} \partial r^{m}}\right|, \\
& E_{(n, m)}=\left|\frac{\partial^{n+m} f(b, d)}{\partial t^{n} \partial r^{m}}\right|, \quad F_{(n, m)}=\left|\frac{\partial^{n+m} f\left(\frac{a+b}{2}, \frac{c+d}{2}\right)}{\partial t^{n} \partial r^{m}}\right|, \quad G_{(n, m)}=\left|\frac{\partial^{n+m} f\left(a, \frac{c+d}{2}\right)}{\partial t^{n} \partial r^{m}}\right|, \\
& H_{(n, m)}=\left|\frac{\partial^{n+m} f\left(\frac{a+b}{2}, c\right)}{\partial t^{n} \partial r^{m}}\right|, \quad J_{(n, m)}=\left|\frac{\partial^{n+m} f\left(\frac{a+b}{2}, d\right)}{\partial t^{n} \partial r^{m}}\right|, \quad I_{(n, m)}=\left|\frac{\partial^{n+m} f\left(b, \frac{c+d}{2}\right)}{\partial t^{n} \partial r^{m}}\right|,
\end{aligned}
$$

where the sums above take 0 , when $m=n=1$ and $m=n=2$ and hence

$$
A^{\prime}=A=\frac{1}{2}\left[\frac{1}{b-a} \int_{a}^{b}[f(x, c)+f(x, d)] d x+\frac{1}{d-c} \int_{c}^{d}[f(a, y)+f(b, y)] d y\right] \text {. }
$$

In what follows $\Delta^{\circ}$ is the interior of $\Delta=[a, b] \times[c, d]$ and $L(\Delta)$ is the space of integrable functions over $\Delta$.

The following two results will be very useful in the sequel of the paper

Theorem 2.1. [18] Let $f: \Delta \rightarrow \mathbb{R}$ be a continuous mapping such that the partial derivatives $\frac{\partial^{k+l} f(., .)}{\partial x^{k} \partial y^{l}}, k=0,1, \ldots, n-1, l=0,1, \ldots, m-1$ exist on $\Delta^{\circ}$ and are continuous on $\Delta$, then

$$
\begin{aligned}
& \int_{a}^{b} \int_{c}^{d} f(t, r) d r d t=(-1)^{m+n} \int_{a}^{b} \int_{c}^{d} K_{n}(x, t) S_{m}(y, r) \frac{\partial^{n+m} f(t, r)}{\partial t^{n} \partial r^{m}} d r d t \\
& +\sum_{k=0}^{n-1} \sum_{l=0}^{m-1} X_{k}(x) Y_{l}(y) \frac{\partial^{k+l} f(x, y)}{\partial x^{k} \partial y^{l}}+(-1)^{m} \sum_{k=0}^{n-1} X_{k}(x) \int_{c}^{d} S_{m}(y, r) \frac{\partial^{k+m} f(x, r)}{\partial x^{k} \partial r^{m}} d r \\
& +(-1)^{n} \sum_{l=0}^{m-1} Y_{l}(y) \int_{a}^{b} K_{n}(x, t) \frac{\partial^{n+l} f(t, y)}{\partial t^{n} \partial y^{l}} d t
\end{aligned}
$$

where, for $(x, y) \in \Delta$, we have

$$
\left\{\begin{array} { r l } 
{ K _ { n } ( x , t ) : = \{ \begin{array} { l } 
{ \frac { ( t - a ) ^ { n } } { n ! } , t \in [ a , x ] } \\
{ \frac { ( t - b ) ^ { n } } { n ! } , t \in ( x , b ] }
\end{array} } \\
{ S _ { m } ( y , r ) : = \{ \begin{array} { l } 
{ \frac { ( r - c ) ^ { m } } { m ! } , r \in [ c , y ] } \\
{ \frac { ( r - d ) ^ { m } } { m ! } , r \in ( y , d ] }
\end{array} \quad \text { and } }
\end{array} \quad \left\{\begin{array}{c}
X_{k}(x):=\frac{(b-x)^{k+1}+(-1)^{k}(x-a)^{k+1}}{(k+1) !} \\
Y_{l}(y):=\frac{(d-y)^{l+1}+(-1)^{l}(y-c)^{l+1}}{(l+1) !}
\end{array} .\right.\right.
$$


Lemma 2.1. [24] Let $f: \Delta \rightarrow \mathbb{R}$, be a continuous mapping such that $\frac{\partial^{m+n} f}{\partial x^{n} \partial y^{m}}$ exists on $\Delta^{\circ}$ and $\frac{\partial^{m+n} f}{\partial x^{n} \partial y^{m}} \in L(\Delta)$ for $m, n \geq 1$, then

$$
\begin{aligned}
& \frac{(b-a)^{n}(d-c)^{m}}{4 n ! m !} \int_{0}^{1} \int_{0}^{1} t^{n-1} r^{m-1}(n-2 t)(m-2 r) \\
& \quad \times \frac{\partial^{n+m} f(t a+(1-t) b, c r+(1-r) d)}{\partial t^{n} \partial r^{m}} d t d r+A^{\prime} \\
& =\frac{f(a, c)+f(a, d)+f(b, c)+f(b, d)}{4}+\frac{1}{(b-a)(d-c)} \int_{a}^{b} \int_{c}^{d} f(x, y) d y d x .
\end{aligned}
$$

Now we prove our main results.

Theorem 2.2. Let $f: \Delta \subseteq[0, \infty)^{2} \rightarrow[0, \infty), a<b, c<d$, be a continuous mapping such that $\frac{\partial^{m+n} f}{\partial t^{n} \partial r^{m}}$ exists on $\Delta^{\circ}$ and $\frac{\partial^{m+n} f}{\partial t^{n} \partial r^{m}} \in L(\Delta)$. If $\left|\frac{\partial^{n+m} f}{\partial t^{n} \partial r^{m}}\right|$ is s-convex on the co-ordinates on $\Delta$ in the second sense, for $m, n \in \mathbb{N}, m, n \geq 2$, then we have the following inequality

$$
\begin{aligned}
& \left|\frac{f(a, c)+f(a, d)+f(b, c)+f(b, d)}{4}+\frac{1}{(b-a)(d-c)} \int_{a}^{b} \int_{c}^{d} f(x, y) d y d x-A^{\prime}\right| \\
& \quad \leq \frac{(b-a)^{n}(d-c)^{m}}{4 n ! m !}\left[L B_{(n, m)}+M C_{(n, m)}+N D_{(n, m)}+R E_{(n, m)}\right],
\end{aligned}
$$

where $s_{1}, s_{2} \in(0,1]$ with $s=\frac{s_{1}+s_{2}}{2}$,

$$
\begin{aligned}
L & =\left[\frac{n(n-1)+s_{1}(n-2)}{\left(n+s_{1}\right)\left(n+s_{1}+1\right)}\right]\left[\frac{m(m-1)+s_{2}(m-2)}{\left(m+s_{2}\right)\left(m+s_{2}+1\right)}\right], \\
M & =\left[\frac{n(n-1)+s_{1}(n-2)}{\left(n+s_{1}\right)\left(n+s_{1}+1\right)}\right]\left[m B\left(m, s_{2}+1\right)-2 B\left(m+1, s_{2}+1\right)\right], \\
N & =\left[\frac{m(m-1)+s_{2}(m-2)}{\left(m+s_{2}\right)\left(m+s_{2}+1\right)}\right]\left[n B\left(n, s_{1}+1\right)-2 B\left(n+1, s_{1}+1\right)\right], \\
R & =\left[n B\left(n, s_{1}+1\right)-2 B\left(n+1, s_{1}+1\right)\right]\left[m B\left(m, s_{2}+1\right)-2 B\left(m+1, s_{2}+1\right)\right],
\end{aligned}
$$

and $B(x, y)=\int_{0}^{1} t^{x-1}(1-t)^{y-1} d t$ is the Euler Beta function.

Proof. Suppose $m, n \geq 2$. By Lemma 2.1, we have

$$
\left|\frac{f(a, c)+f(a, d)+f(b, c)+f(b, d)}{4}+\frac{1}{(b-a)(d-c)} \int_{a}^{b} \int_{c}^{d} f(x, y) d y d x-A^{\prime}\right|
$$

$$
\begin{aligned}
\leq & \frac{(b-a)^{n}(d-c)^{m}}{4 n ! m !} \int_{0}^{1} \int_{0}^{1} t^{n-1} r^{m-1}(n-2 t)(m-2 r) \\
& \times\left|\frac{\partial^{n+m} f(t a+(1-t) b, c r+(1-r) d)}{\partial t^{n} \partial r^{m}}\right| d t d r .
\end{aligned}
$$


By $s$-convexity of $\left|\frac{\partial^{m+n} f}{\partial t^{n} \partial s^{m}}\right|$ on the co-ordinates on $\Delta$, we get that

$$
\int_{0}^{1} \int_{0}^{1} t^{n-1} r^{m-1}(n-2 t)(m-2 r) \times\left|\frac{\partial^{n+m} f(t a+(1-t) b, c r+(1-r) d)}{\partial t^{n} \partial r^{m}}\right| d t d r
$$

$$
\begin{aligned}
\leq & B_{(n, m)} \int_{0}^{1} \int_{0}^{1} t^{n+s_{1}-1} r^{m+s_{2}-1}(n-2 t)(m-2 r) d r d t \\
& +C_{(n, m)} \int_{0}^{1} \int_{0}^{1} t^{n+s_{1}-1} r^{m-1}(1-r)^{s_{2}}(n-2 t)(m-2 r) d r d t \\
& +E_{(n, m)} \int_{0}^{1} \int_{0}^{1} t^{n-1}(1-t)^{s_{1}}(n-2 t) r^{m-1}(1-r)^{s_{2}}(m-2 r) d r d t \\
& +D_{(n, m)} \int_{0}^{1} \int_{0}^{1} t^{n-1} r^{m+s_{2}-1}(1-t)^{s_{1}}(n-2 t)(m-2 r) d r d t .
\end{aligned}
$$

Since

$$
\begin{aligned}
& \int_{0}^{1} \int_{0}^{1} t^{n+s_{1}-1} r^{m+s_{2}-1}(n-2 t)(m-2 r) d r d t \\
& =\int_{0}^{1} t^{n+s_{1}-1}(n-2 t) d t \int_{0}^{1} r^{m+s_{2}-1}(m-2 r) d r \\
& =\left[\frac{n(n-1)+s_{1}(n-2)}{\left(n+s_{1}\right)\left(n+s_{1}+1\right)}\right]\left[\frac{m(m-1)+s_{2}(m-2)}{\left(m+s_{2}\right)\left(m+s_{2}+1\right)}\right] .
\end{aligned}
$$

Analogously,

$$
\begin{aligned}
& \int_{0}^{1} \int_{0}^{1} t^{n+s_{1}-1} r^{m-1}(1-r)^{s_{2}}(n-2 t)(m-2 r) d r d t \\
= & {\left[\frac{n(n-1)+s_{1}(n-2)}{\left(n+s_{1}\right)\left(n+s_{1}+1\right)}\right]\left[m B\left(m, s_{2}+1\right)-2 B\left(m+1, s_{2}+1\right)\right] } \\
& \int_{0}^{1} \int_{0}^{1} t^{n-1} r^{m+s_{2}-1}(1-t)^{s_{1}}(n-2 t)(m-2 r) d r d t \\
= & {\left[\frac{m(m-1)+s_{2}(m-2)}{\left(m+s_{2}\right)\left(m+s_{2}+1\right)}\right]\left[n B\left(n, s_{1}+1\right)-2 B\left(n+1, s_{1}+1\right)\right] }
\end{aligned}
$$

and

$$
\int_{0}^{1} \int_{0}^{1} t^{n-1}(1-t)^{s_{1}}(n-2 t) r^{m-1}(1-r)^{s_{2}}(m-2 r) d r d t
$$

From (2.4)-(2.8) in (2.3), we get the required inequality. This completes the proof of the theorem. 
Theorem 2.3. Let $f: \Delta \subset[0, \infty) \times[0, \infty) \rightarrow[0, \infty), a<b, c<d$, be a continuous mapping such that $\frac{\partial^{m+n} f}{\partial t^{n} \partial r^{m}}$ exists on $\Delta^{\circ}$ and $\frac{\partial^{m+n} f}{\partial t^{n} \partial r^{m}} \in L(\Delta)$. If $\left|\frac{\partial^{n+m} f}{\partial t^{n} \partial r^{m}}\right|^{q}, q \geq 1$, is $s$-convex on the co-ordinates on $\Delta, m, n \in \mathbb{N}, m, n \geq 2$, then

$$
\left|\frac{f(a, c)+f(a, d)+f(b, c)+f(b, d)}{4}+\frac{1}{(b-a)(d-c)} \int_{a}^{b} \int_{c}^{d} f(x, y) d y d x-A^{\prime}\right|
$$

$$
\begin{aligned}
\leq & \frac{(b-a)^{n}(d-c)^{m}}{4 n ! m !}\left(\frac{(n-1)(m-1)}{(n+1)(m+1)}\right)^{1-1 / q} \\
& \times \sqrt[q]{L B_{(n, m)}^{q}+M D_{(n, m)}^{q}+N C_{(n, m)}^{q}+R E_{(n, m)}^{q}},
\end{aligned}
$$

where $s_{1}, s_{2} \in(0,1]$ with $s=\frac{s_{1}+s_{2}}{2}$ and $L, M, N, R$ and $B(x, y)$ are as defined in Theorem 2.2.

Proof. The case $q=1$ is the Theorem 2.2. Suppose $q>1$, then by Lemma 2.1 and the power mean inequality, we have

$$
\left|\frac{f(a, c)+f(a, d)+f(b, c)+f(b, d)}{4}+\frac{1}{(b-a)(d-c)} \int_{a}^{b} \int_{c}^{d} f(x, y) d y d x-A^{\prime}\right|
$$

$$
\begin{aligned}
\leq & \frac{(b-a)^{n}(d-c)^{m}}{4 n ! m !}\left\{\int_{0}^{1} \int_{0}^{1} t^{n-1} r^{m-1}(n-2 t)(m-2 r) d r d t\right\}^{1-1 / q} \\
& \times\left\{\int_{0}^{1} \int_{0}^{1} t^{n-1} r^{m-1}(n-2 t)(m-2 r)\right. \\
& \left.\times\left|\frac{\partial^{n+m} f(t a+(1-t) b, c r+(1-r) d)}{\partial t^{n} \partial r^{m}}\right|^{q} d t d r\right\}^{1 / q} .
\end{aligned}
$$

By the similar arguments used to obtain (2.2) and the fact

$$
\int_{0}^{1} \int_{0}^{1} t^{n-1} r^{m-1}(n-2 t)(m-2 r) d r d t=\frac{(n-1)(m-1)}{(n+1)(m+1)}
$$

we get (2.9). This completes the proof of the theorem.

Theorem 2.4. Let $f: \Delta \subset[0, \infty) \times[0, \infty) \rightarrow[0, \infty), a<b, c<d$, be a continuous mapping such that $\frac{\partial^{m+n} f}{\partial t^{n} \partial r^{m}}$ exist on $\Delta^{\circ}$ and $\frac{\partial^{m+n} f}{\partial t^{n} \partial r^{m}} \in L(\Delta)$. If $\left|\frac{\partial^{n+m} f}{\partial t^{n} \partial s^{m}}\right|^{q}, q \geq 1$, is $s$-convex on the co-ordinates on $\Delta, s_{1}, s_{2} \in(0,1]$ with $s=\frac{s_{1}+s_{2}}{2}, m, n \in \mathbb{N}, m, n \geq 1$. 
Then

$$
\begin{aligned}
\mid & -\sum_{k=0}^{n-1} \sum_{l=0}^{m-1} \frac{\left[1+(-1)^{k}\right]\left[1+(-1)^{l}\right]}{2^{k+l+2}} \frac{(b-a)^{k}(d-c)^{l}}{(k+1) !(l+1) !} \frac{\partial^{k+l} f\left(\frac{a+b}{2}, \frac{c+d}{2}\right)}{\partial x^{k} \partial y^{l}} \\
& +\frac{1}{(b-a)(d-c)} \int_{a}^{b} \int_{c}^{d} f(t, r) d r d t \\
& +\frac{(-1)^{m+1}}{(d-c) m !} \sum_{k=0}^{n-1} \frac{\left[1+(-1)^{k}\right](b-a)^{k}}{2^{k+1}(k+1) !} \int_{c}^{d} Q(r) \frac{\partial^{k+m} f\left(\frac{a+b}{2}, r\right)}{\partial x^{k} \partial r^{m}} d r \\
& +\frac{(-1)^{n+1}}{(b-a) n !} \sum_{l=0}^{m-1}\left[1+(-1)^{l}\right](d-c)^{l} \\
2^{l+1}(l+1) ! & \int_{a}^{b} P(t) \frac{\partial^{n+l} f\left(t, \frac{c+d}{2}\right)}{\partial t^{n} \partial y^{l}} d t \mid \\
\leq & \frac{1}{4 n ! m !}\left(\frac{4}{(n+1)(m+1)}\right)^{1-\frac{1}{q}}\left(\frac{b-a}{2}\right)^{n}\left(\frac{d-c}{2}\right)^{m} \\
& \times\left[\left(B_{(n, m)}^{q}+C_{(n, m)}^{q}+D_{(n, m)}^{q}+E_{(n, m)}^{q}\right) B\left(n+1, s_{1}+1\right) B\left(m+1, s_{2}+1\right)\right. \\
& +\frac{2\left(G_{(n, m)}^{q}+I_{(n, m)}^{q}\right) B\left(n+1, s_{1}+1\right)}{m+s_{2}+1}+\frac{2\left(H_{(n, m)}^{q}+J_{(n, m)}^{q}\right) B\left(m+1, s_{2}+1\right)}{n+s_{1}+1} \\
& \left.+\frac{4 F_{(n, m)}^{q}}{\left(n+s_{1}+1\right)\left(m+s_{2}+1\right)}\right]^{\frac{1}{q}},
\end{aligned}
$$

where

$$
P(t):=\left\{\begin{array}{c}
(t-a)^{n}, t \in\left[a, \frac{a+b}{2}\right] \\
(t-b)^{n}, t \in\left(\frac{a+b}{2}, b\right]
\end{array} \quad \text { and } \quad Q(r):=\left\{\begin{array}{c}
(r-c)^{m}, r \in\left[c, \frac{c+d}{2}\right] \\
(r-d)^{m}, r \in\left(\frac{c+d}{2}, d\right]
\end{array} .\right.\right.
$$

Proof. By letting $x \mapsto \frac{a+b}{2}$ and $y \mapsto \frac{c+d}{2}$ in Theorem 2.1 and using the properties of the absolute value, we obtain

$$
\begin{aligned}
\mid & -\sum_{k=0}^{n-1} \sum_{l=0}^{m-1} \frac{\left[1+(-1)^{k}\right]\left[1+(-1)^{l}\right]}{2^{k+l+2}} \frac{(b-a)^{k}(d-c)^{l}}{(k+1) !(l+1) !} \frac{\partial^{k+l} f\left(\frac{a+b}{2}, \frac{c+d}{2}\right)}{\partial x^{k} \partial y^{l}} \\
& +\frac{1}{(b-a)(d-c)} \int_{a}^{b} \int_{c}^{d} f(t, r) d r d t \\
& +\frac{(-1)^{m+1}}{(d-c) m !} \sum_{k=0}^{n-1} \frac{\left[1+(-1)^{k}\right](b-a)^{k}}{2^{k+1}(k+1) !} \int_{c}^{d} Q(r) \frac{\partial^{k+m} f\left(\frac{a+b}{2}, r\right)}{\partial x^{k} \partial r^{m}} d r
\end{aligned}
$$




$$
\begin{aligned}
& +\frac{(-1)^{n+1}}{(b-a) n !} \sum_{l=0}^{m-1} \frac{\left[1+(-1)^{l}\right](d-c)^{l}}{2^{l+1}(l+1) !} \int_{a}^{b} P(t) \frac{\partial^{n+l} f\left(t, \frac{c+d}{2}\right)}{\partial t^{n} \partial y^{l}} d t \mid \\
\leq & \frac{1}{(b-a)(d-c) m ! n !} \int_{a}^{b} \int_{c}^{d}|P(t)||Q(r)|\left|\frac{\partial^{n+m} f(t, r)}{\partial t^{n} \partial r^{m}}\right| d r d t .
\end{aligned}
$$

By the power mean inequality for double integrals, we have

$$
\int_{a}^{b} \int_{c}^{d}|P(t)||Q(r)|\left|\frac{\partial^{n+m} f(t, r)}{\partial t^{n} \partial r^{m}}\right| d r d t
$$

$$
\begin{aligned}
\leq & \left(\int_{a}^{b} \int_{c}^{d}|P(t)||Q(r)| d r d t\right)^{1-\frac{1}{q}}\left(\left.\int_{a}^{b} \int_{c}^{d}|P(t)||Q(r)| \frac{\partial^{n+m} f(t, r)}{\partial t^{n} \partial r^{m}}\right|^{q} d r d t\right)^{\frac{1}{q}} \\
= & \left(\int_{a}^{b} \int_{c}^{d}|P(t)||Q(r)| d r d t\right)^{1-\frac{1}{q}}\left[\int_{a}^{\frac{a+b}{2}} \int_{c}^{\frac{c+d}{2}}(t-a)^{n}(r-c)^{m}\left|\frac{\partial^{n+m} f(t, r)}{\partial t^{n} \partial r^{m}}\right|^{q} d r d t\right. \\
& +\int_{\frac{a+b}{2}}^{b} \int_{c}^{\frac{c+d}{2}}(b-t)^{n}(r-c)^{m}\left|\frac{\partial^{n+m} f(t, r)}{\partial t^{n} \partial r^{m}}\right|^{q} d r d t \\
& +\int_{a}^{\frac{a+b}{2}} \int_{\frac{c+d}{2}}^{d}(t-a)^{n}(d-r)^{m}\left|\frac{\partial^{n+m} f(t, r)}{\partial t^{n} \partial r^{m}}\right|^{q} d r d t \\
& \left.+\int_{\frac{a+b}{2}}^{b} \int_{\frac{c+d}{2}}^{d}(b-t)^{n}(d-r)^{m}\left|\frac{\partial^{n+m} f(t, r)}{\partial t^{n} \partial r^{m}}\right|^{q} d r d t\right]^{\frac{1}{q}} .
\end{aligned}
$$

Now we calculate each integral in (2.13). Since $t=\left(\frac{\frac{a+b}{2}-t}{\frac{a+b}{2}-a}\right) a+\left(\frac{t-a}{\frac{a+b}{2}-a}\right) \frac{a+b}{2}$ and $r=\left(\frac{\frac{c+d}{2}-r}{\frac{c+d}{2}-c}\right) c+\left(\frac{r-c}{\frac{c+d}{2}-c}\right) \frac{c+d}{2}$. By the co-ordinated $s$-convexity of $\left|\frac{\partial^{n+m} f}{\partial t^{n} \partial s^{m}}\right|^{q}$, we have

$$
\begin{aligned}
& \int_{a}^{\frac{a+b}{2}} \int_{c}^{\frac{c+d}{2}}(t-a)^{n}(r-c)^{m}\left|\frac{\partial^{n+m} f(t, r)}{\partial t^{n} \partial r^{m}}\right|^{q} d r d t \leq\left(\frac{2}{b-a}\right)^{s_{1}}\left(\frac{2}{d-c}\right)^{s_{2}} \\
& \times\left[B_{(n, m)}^{q} \int_{a}^{\frac{a+b}{2}} \int_{c}^{\frac{c+d}{2}}(t-a)^{n}(r-c)^{m}\left(\frac{a+b}{2}-t\right)^{s_{1}}\left(\frac{c+d}{2}-r\right)^{s_{2}} d r d t\right. \\
& \quad+G_{(n, m)}^{q} \int_{a}^{\frac{a+b}{2}} \int_{c}^{\frac{c+d}{2}}(t-a)^{n}\left(\frac{a+b}{2}-t\right)^{s_{1}}(r-c)^{s_{2}+m} d r d t \\
& \quad+H_{(n, m)}^{q} \int_{a}^{\frac{a+b}{2}} \int_{c}^{\frac{c+d}{2}}(t-a)^{s_{1}+n}\left(\frac{c+d}{2}-r\right)^{s_{2}}(r-c)^{m} d r d t \\
& \left.\quad+F_{(n, m)}^{q} \int_{a}^{\frac{a+b}{2}} \int_{c}^{\frac{c+d}{2}}(t-a)^{s_{1}+n}(r-c)^{s_{2}+m} d r d t\right] .
\end{aligned}
$$


Now by the change of variables $u=t-a, v=r-c$ and then by the change of variables $x=\frac{2 u}{b-a}, y=\frac{2 v}{d-c}$, we get that

$$
\begin{aligned}
& \left(\frac{2}{b-a}\right)^{s_{1}}\left(\frac{2}{d-c}\right)^{s_{2}} \times \int_{a}^{\frac{a+b}{2}} \int_{c}^{\frac{c+d}{2}}(t-a)^{n}(r-c)^{m}\left(\frac{a+b}{2}-t\right)^{s_{1}}\left(\frac{c+d}{2}-r\right)^{s_{2}} d r d t \\
& =\left(\frac{2}{b-a}\right)^{s_{1}}\left(\frac{2}{d-c}\right)^{s_{2}} \int_{0}^{\frac{b-a}{2}} u^{n}\left(\frac{b-a}{2}-u\right)^{s_{1}} d u \int_{0}^{\frac{d-c}{2}} v^{m}\left(\frac{d-c}{2}-v\right)^{s_{2}} d v \\
& =\int_{0}^{\frac{b-a}{2}} u^{n}\left(1-\frac{2 u}{b-a}\right)^{s_{1}} d u \int_{0}^{\frac{d-c}{2}} v^{m}\left(1-\frac{2 v}{d-c}\right)^{s_{2}} d v \\
& =\left(\frac{b-a}{2}\right)^{n+1}\left(\frac{d-c}{2}\right)^{m+1} \int_{0}^{1} x^{n}(1-x)^{s_{1}} d x \int_{0}^{1} y^{m}(1-y)^{s_{2}} d y \\
& =\left(\frac{b-a}{2}\right)^{n+1}\left(\frac{d-c}{2}\right)^{m+1} B\left(n+1, s_{1}+1\right) B\left(m+1, s_{2}+1\right) .
\end{aligned}
$$

Similarly,

$$
\begin{aligned}
& \left(\frac{2}{b-a}\right)^{s_{1}}\left(\frac{2}{d-c}\right)^{s_{2}} \int_{a}^{\frac{a+b}{2}} \int_{c}^{\frac{c+d}{2}}(t-a)^{n}\left(\frac{a+b}{2}-t\right)^{s_{1}}(r-c)^{s_{2}+m} d r d t \\
& =\frac{\left(\frac{b-a}{2}\right)^{n+1}\left(\frac{d-c}{2}\right)^{m+1} B\left(n+1, s_{1}+1\right)}{m+s_{2}+1} \\
& \left(\frac{2}{b-a}\right)^{s_{1}}\left(\frac{2}{d-c}\right)^{s_{2}} \int_{a}^{\frac{a+b}{2}} \int_{c}^{\frac{c+d}{2}}(t-a)^{s_{1}+n}\left(\frac{c+d}{2}-r\right)^{s_{2}}(r-c)^{m} d r d t \\
& =\frac{\left(\frac{b-a}{2}\right)^{n+1}\left(\frac{d-c}{2}\right)^{m+1} B\left(m+1, s_{2}+1\right)}{n+s_{1}+1}
\end{aligned}
$$

and

$$
\begin{aligned}
& \left(\frac{2}{b-a}\right)^{s_{1}}\left(\frac{2}{d-c}\right)^{s_{2}} \int_{a}^{\frac{a+b}{2}} \int_{c}^{\frac{c+d}{2}}(t-a)^{s_{1}+n}(r-c)^{s_{2}+m} d r d t \\
& =\frac{\left(\frac{b-a}{2}\right)^{n+1}\left(\frac{d-c}{2}\right)^{m+1}}{\left(n+s_{1}+1\right)\left(m+s_{2}+1\right)} .
\end{aligned}
$$

Using (2.15)-(2.18) in (2.14), we obtain

$$
\begin{aligned}
& \int_{a}^{\frac{a+b}{2}} \int_{c}^{\frac{c+d}{2}}(t-a)^{n}(r-c)^{m}\left|\frac{\partial^{n+m} f(t, r)}{\partial t^{n} \partial r^{m}}\right|^{q} d r d t \leq\left(\frac{b-a}{2}\right)^{n+1}\left(\frac{d-c}{2}\right)^{m+1} \\
& \times\left[B_{(n, m)}^{q} B\left(n+1, s_{1}+1\right) B\left(m+1, s_{2}+1\right)+\frac{G_{(n, m)}^{q} B\left(n+1, s_{1}+1\right)}{m+s_{2}+1}\right.
\end{aligned}
$$




$$
\left.+\frac{H_{(n, m)}^{q} B\left(m+1, s_{2}+1\right)}{n+s_{1}+1}+\frac{F_{(n, m)}^{q}}{\left(n+s_{1}+1\right)\left(m+s_{2}+1\right)}\right] .
$$

Analogously,

$$
\begin{gathered}
\int_{\frac{a+b}{2}}^{b} \int_{c}^{\frac{c+d}{2}}(b-t)^{n}(r-c)^{m}\left|\frac{\partial^{n+m} f(t, r)}{\partial t^{n} \partial r^{m}}\right|^{q} d r d t \leq\left(\frac{b-a}{2}\right)^{n+1}\left(\frac{d-c}{2}\right)^{m+1} \\
\times\left[\frac{H_{(n, m)}^{q} B\left(m+1, s_{2}+1\right)}{n+s_{1}+1}+D_{(n, m)}^{q} B\left(n+1, s_{1}+1\right) B\left(m+1, s_{2}+1\right)\right. \\
\left.+\frac{F_{(n, m)}^{q}}{\left(n+s_{1}+1\right)\left(m+s_{2}+1\right)}+\frac{I_{(n, m)}^{q} B\left(n+1, s_{1}+1\right)}{m+s_{2}+1}\right], \\
\int_{a}^{\frac{a+b}{2}} \int_{\frac{c+d}{2}}^{d}(t-a)^{n}(d-r)^{m}\left|\frac{\partial^{n+m} f(t, r)}{\partial t^{n} \partial r^{m}}\right|^{q} d r d t \\
\leq\left(\frac{b-a}{2}\right)^{n+1}\left(\frac{d-c}{2}\right)^{m+1}\left[\frac{G_{(n, m)}^{q} B\left(n+1, s_{1}+1\right)}{m+s_{2}+1}\right. \\
+C_{(n, m)}^{q} B\left(n+1, s_{1}+1\right) B\left(m+1, s_{2}+1\right) \\
\left.+\frac{J_{(n, m)}^{q} B\left(m+1, s_{2}+1\right)}{n+s_{1}+1}+\frac{F_{(n, m)}^{q}}{\left(n+s_{1}+1\right)\left(m+s_{2}+1\right)}\right]
\end{gathered}
$$

and

$$
\int_{\frac{a+b}{2}}^{b} \int_{\frac{c+d}{2}}^{d}(b-t)^{n}(d-r)^{m}\left|\frac{\partial^{n+m} f(t, r)}{\partial t^{n} \partial r^{m}}\right|^{q} d r d t
$$

$$
\begin{aligned}
\leq & \left(\frac{b-a}{2}\right)^{n+1}\left(\frac{d-c}{2}\right)^{m+1}\left[\frac{F_{(n, m)}^{q}}{\left(n+s_{1}+1\right)\left(m+s_{2}+1\right)}+\frac{I_{(n, m)}^{q} B\left(n+1, s_{1}+1\right)}{m+s_{2}+1}\right. \\
& \left.+\frac{J_{(n, m)}^{q} B\left(m+1, s_{2}+1\right)}{n+s_{1}+1}+E_{(n, m)}^{q} B\left(n+1, s_{1}+1\right) B\left(m+1, s_{2}+1\right)\right] .
\end{aligned}
$$

It is not difficult to observe that

$$
\int_{a}^{b} \int_{c}^{d}|P(t)||Q(r)| d r d t=\frac{4}{(n+1)(m+1)}\left(\frac{b-a}{2}\right)^{n+1}\left(\frac{d-c}{2}\right)^{m+1} .
$$

From (2.12)-(2.23), we get the desired inequality. The proof of the Theorem for $q=1$ is the same. This completes the proof.

Some results can be deduced from the inequalities (2.9) and (2.12) as follows. Letting $s_{1}=s_{2}=1$ in Theorem 2.3 gives the following corollary. 
Corollary 2.1. Let $f: \Delta \subset[0, \infty) \times[0, \infty) \rightarrow[0, \infty), a<b, c<d$, be a continuous mapping such that $\frac{\partial^{m+n} f}{\partial t^{n} \partial r^{m}}$ exists on $\Delta^{\circ}$ and $\frac{\partial^{m+n} f}{\partial t^{n} \partial r^{m}} \in L(\Delta)$. If $\left|\frac{\partial^{n+m} f}{\partial t^{n} \partial r^{m}}\right|^{q}, q \geq 1$, is convex on the co-ordinates on $\Delta, m, n \in \mathbb{N}, m, n \geq 2$, then

$$
\left|\frac{f(a, c)+f(a, d)+f(b, c)+f(b, d)}{4}+\frac{1}{(b-a)(d-c)} \int_{a}^{b} \int_{c}^{d} f(x, y) d y d x-A^{\prime}\right|
$$

$$
\begin{aligned}
\leq & \frac{(b-a)^{n}(d-c)^{m}(n-1)^{1-1 / q}(m-1)^{1-1 / q}}{4(n+1) !(m+1) !(n+2)^{1 / q}(m+2)^{1 / q}}\left[\left(m^{2}-2\right)\left(n^{2}-2\right) B_{(n, m)}^{q}\right. \\
& \left.+m\left(n^{2}-2\right) C_{(n, m)}^{q}+n\left(m^{2}-2\right) D_{(n, m)}^{q}+n m E_{(n, m)}^{q}\right]^{\frac{1}{q}} .
\end{aligned}
$$

Corollary 2.2. Under the assumptions of Corollary 2.1 with $m=n=2$, we have

$$
\begin{aligned}
& \left|\frac{f(a, c)+f(a, d)+f(b, c)+f(b, d)}{4}+\frac{1}{(b-a)(d-c)} \int_{a}^{b} \int_{c}^{d} f(x, y) d y d x-A^{\prime}\right| \\
& \leq \frac{(b-a)^{2}(d-c)^{2}}{9 \cdot 2^{\frac{2}{q}+4}} \sqrt[q]{\left|\frac{\partial^{4} f(a, c)}{\partial t^{2} \partial r^{2}}\right|^{q}+\left|\frac{\partial^{4} f(b, c)}{\partial t^{2} \partial r^{2}}\right|^{q}+\left|\frac{\partial^{4} f(a, d)}{\partial t^{2} \partial r^{2}}\right|^{q}+\left|\frac{\partial^{4} f(b, d)}{\partial t^{2} \partial r^{2}}\right|^{q}} .
\end{aligned}
$$

The following corollary is a special case of Theorem 2.4 for $s_{1}=s_{2}=1$.

Corollary 2.3. Let $f: \Delta \subset[0, \infty) \times[0, \infty) \rightarrow[0, \infty), a<b, c<d$, be a continuous mapping such that $\frac{\partial^{m+n} f}{\partial t^{n} \partial r^{m}}$ exist on $\Delta^{\circ}$ and $\frac{\partial^{m+n} f}{\partial t^{n} \partial r^{m}} \in L(\Delta)$. If $\left|\frac{\partial^{n+m} f}{\partial t^{n} \partial s^{m}}\right|^{q}, q \geq 1$, is convex on the co-ordinates on $\Delta, m, n \in \mathbb{N}, m, n \geq 1$. Then

$$
\begin{array}{|l}
\mid-\sum_{k=0}^{n-1} \sum_{l=0}^{m-1} \frac{\left[1+(-1)^{k}\right]\left[1+(-1)^{l}\right]}{2^{k+l+2}} \frac{(b-a)^{k}(d-c)^{l}}{(k+1) !(l+1) !} \frac{\partial^{k+l} f\left(\frac{a+b}{2}, \frac{c+d}{2}\right)}{\partial x^{k} \partial y^{l}} \\
+\frac{1}{(b-a)(d-c)} \int_{a}^{b} \int_{c}^{d} f(t, r) d r d t \\
+\frac{(-1)^{m+1}}{(d-c) m !} \sum_{k=0}^{n-1} \frac{\left[1+(-1)^{k}\right](b-a)^{k}}{2^{k+1}(k+1) !} \int_{c}^{d} Q(r) \frac{\partial^{k+m} f\left(\frac{a+b}{2}, r\right)}{\partial x^{k} \partial r^{m}} d r \\
\quad+\frac{(-1)^{n+1}}{(b-a) n !} \sum_{l=0}^{m-1} \frac{\left[1+(-1)^{l}\right](d-c)^{l}}{2^{l+1}(l+1) !} \int_{a}^{b} P(t) \frac{\partial^{n+l} f\left(t, \frac{c+d}{2}\right)}{\partial t^{n} \partial y^{l}} d t \mid
\end{array}
$$




$$
\begin{aligned}
\leq & \frac{(b-a)^{n}(d-c)^{m}}{2^{m+n+\frac{2}{q}}(n+1) !(m+1) !}\left[\frac{B_{(n, m)}^{q}+C_{(n, m)}^{q}+D_{(n, m)}^{q}+E_{(n, m)}^{q}}{(n+2)(m+2)}\right. \\
& +\frac{2(m+1)\left(G_{(n, m)}^{q}+I_{(n, m)}^{q}\right)}{(n+2)(m+2)}+\frac{2(n+1)\left(H_{(n, m)}^{q}+J_{(n, m)}^{q}\right)}{(n+2)(m+2)} \\
& \left.+\frac{4(n+1)(m+1) F_{(n, m)}^{q}}{(n+2)(m+2)}\right]^{\frac{1}{q}},
\end{aligned}
$$

where $P(t)$ and $Q(r)$ are as defined in Theorem 2.4.

The following corollary is a special case of Theorem 2.4 for $s_{1}=s_{2}=1$ and $m=n=1$, which gives tighter estimate than those from [23, Theorem 4, page 8].

Corollary 2.4. Under the assumptions of Corollary 2.3 with $m=n=1$, we have

$$
\begin{aligned}
& \mid \frac{1}{(b-a)(d-c)} \int_{a}^{b} \int_{c}^{d} f(t, r) d r d t+f\left(\frac{a+b}{2}, \frac{c+d}{2}\right) \\
& \quad-\frac{1}{2(d-c)} \int_{c}^{d} f\left(\frac{a+b}{2}, r\right) d r-\frac{1}{2(b-a)} \int_{a}^{b} f\left(t, \frac{c+d}{2}\right) d t \mid \\
& \leq \frac{(b-a)(d-c)}{2^{4+\frac{2}{q}}}\left[\frac{B_{(1,1)}^{q}+C_{(1,1)}^{q}+D_{(1,1)}^{q}+E_{(1,1)}^{q}}{9}\right. \\
& \left.\quad+\frac{4\left(G_{(1,1)}^{q}+I_{(1,1)}^{q}\right)}{9}+\frac{4\left(H_{(1,1)}^{q}+J_{(1,1)}^{q}\right)}{9}+\frac{8 F_{(1,1)}^{q}}{9}\right]^{\frac{1}{q}},
\end{aligned}
$$

where $P(t)$ and $Q(r)$ are as defined in Theorem 2.4.

It is easy to see that, when $\left|\frac{\partial^{n+m} f}{\partial t^{n} \partial s^{m}}\right|^{q}, q \geq 1$, is convex on the co-ordinates on $\Delta$, $m, n \in \mathbb{N}, m, n \geq 1$, then

$$
\begin{aligned}
& 2\left(G_{(n, m)}^{q}+I_{(n, m)}^{q}\right) \leq B_{(n, m)}^{q}+C_{(n, m)}^{q}+D_{(n, m)}^{q}+E_{(n, m)}^{q}, \\
& 2\left(H_{(n, m)}^{q}+J_{(n, m)}^{q}\right) \leq B_{(n, m)}^{q}+C_{(n, m)}^{q}+D_{(n, m)}^{q}+E_{(n, m)}^{q}
\end{aligned}
$$

and

$$
4 F_{(n, m)}^{q} \leq B_{(n, m)}^{q}+C_{(n, m)}^{q}+D_{(n, m)}^{q}+E_{(n, m)}^{q} .
$$

Substituting these inequalities in Corollary 2.3, we get the following corollary which is [24, Theorem 2.3, page 12].

Corollary 2.5. Let $f: \Delta \subset[0, \infty) \times[0, \infty) \rightarrow[0, \infty), a<b, c<d$, be a continuous mapping such that $\frac{\partial^{m+n} f}{\partial t^{n} \partial r^{m}}$ exist on $\Delta^{\circ}$ and $\frac{\partial^{m+n} f}{\partial t^{n} \partial r^{m}} \in L(\Delta)$. If $\left|\frac{\partial^{n+m} f}{\partial t^{n} \partial s^{m}}\right|^{q}, q \geq 1$, is 
convex on the co-ordinates on $\Delta, m, n \in \mathbb{N}, m, n \geq 1$. Then

$$
\begin{aligned}
& \mid-\sum_{k=0}^{n-1} \sum_{l=0}^{m-1} \frac{\left[1+(-1)^{k}\right]\left[1+(-1)^{l}\right]}{2^{k+l+2}} \frac{(b-a)^{k}(d-c)^{l}}{(k+1) !(l+1) !} \frac{\partial^{k+l} f\left(\frac{a+b}{2}, \frac{c+d}{2}\right)}{\partial x^{k} \partial y^{l}} \\
& +\frac{1}{(b-a)(d-c)} \int_{a}^{b} \int_{c}^{d} f(t, r) d r d t \\
& +\frac{(-1)^{m+1}}{(d-c) m !} \sum_{k=0}^{n-1} \frac{\left[1+(-1)^{k}\right](b-a)^{k}}{2^{k+1}(k+1) !} \int_{c}^{d} Q(r) \frac{\partial^{k+m} f\left(\frac{a+b}{2}, r\right)}{\partial x^{k} \partial r^{m}} d r \\
& +\frac{(-1)^{n+1}}{(b-a) n !} \sum_{l=0}^{m-1} \frac{\left[1+(-1)^{l}\right](d-c)^{l}}{2^{l+1}(l+1) !} \int_{a}^{b} P(t) \frac{\partial^{n+l} f\left(t, \frac{c+d}{2}\right)}{\partial t^{n} \partial y^{l}} d t \\
& \leq \frac{(b-a)^{n}(d-c)^{m}}{2^{m+n+\frac{2}{q}}(n+1) !(m+1) !} \sqrt[q]{B_{(n, m)}^{q}+C_{(n, m)}^{q}+D_{(n, m)}^{q}+E_{(n, m)}^{q}},
\end{aligned}
$$

where $P(t)$ and $Q(r)$ are as defined in Theorem 2.4.

A different approach leads us to the following result.

Theorem 2.5. Let $f: \Delta \subset[0, \infty) \times[0, \infty) \rightarrow[0, \infty), a<b, c<d$, be a continuous mapping such that $\frac{\partial^{m+n} f}{\partial t^{n} \partial r^{m}}$ exist on $\Delta^{\circ}$ and $\frac{\partial^{m+n} f}{\partial t^{n} \partial r^{m}} \in L(\Delta)$. If $\left|\frac{\partial^{n+m} f}{\partial t^{n} \partial s^{m}}\right|^{q}, q \geq 1$, is $s$-convex on the co-ordinates on $\Delta, s_{1}, s_{2} \in(0,1]$ with $s=\frac{s_{1}+s_{2}}{2}, m, n \in \mathbb{N}, m, n \geq 1$. Then

$$
\begin{aligned}
\mid & -\sum_{k=0}^{n-1} \sum_{l=0}^{m-1} \frac{\left[1+(-1)^{k}\right]\left[1+(-1)^{l}\right]}{2^{k+l+2}} \frac{(b-a)^{k}(d-c)^{l}}{(k+1) !(l+1) !} \frac{\partial^{k+l} f\left(\frac{a+b}{2}, \frac{c+d}{2}\right)}{\partial x^{k} \partial y^{l}} \\
& +\frac{1}{(b-a)(d-c)} \int_{a}^{b} \int_{c}^{d} f(t, r) d r d t \\
& +\frac{(-1)^{m+1}}{(d-c) m !} \sum_{k=0}^{n-1} \frac{\left[1+(-1)^{k}\right](b-a)^{k}}{2^{k+1}(k+1) !} \int_{c}^{d} Q(r) \frac{\partial^{k+m} f\left(\frac{a+b}{2}, r\right)}{\partial x^{k} \partial r^{m}} d r \\
& +\frac{(-1)^{n+1}}{(b-a) n !} \sum_{l=0}^{m-1} \frac{\left[1+(-1)^{l}\right](d-c)^{l}}{2^{l+1}(l+1) !} \int_{a}^{b} P(t) \frac{\partial^{n+l} f\left(t, \frac{c+d}{2}\right)}{\partial t^{n} \partial y^{l}} d t \mid \\
\leq & \frac{1}{4 n ! m !}\left(\frac{1}{(n+1)(m+1)}\right)^{1-\frac{1}{q}}\left(\frac{b-a}{2}\right)^{n}\left(\frac{d-c}{2}\right)^{m} \times
\end{aligned}
$$




$$
\begin{aligned}
& \times\left\{\left[B_{(n, m)}^{q} B\left(n+1, s_{1}+1\right) B\left(m+1, s_{2}+1\right)+\frac{G_{(n, m)}^{q} B\left(n+1, s_{1}+1\right)}{m+s_{2}+1}\right.\right. \\
& \left.+\frac{H_{(n, m)}^{q} B\left(m+1, s_{2}+1\right)}{n+s_{1}+1}+\frac{F_{(n, m)}^{q}}{\left(n+s_{1}+1\right)\left(m+s_{2}+1\right)}\right]^{\frac{1}{q}} \\
& +\left[\frac{H_{(n, m)}^{q} B\left(m+1, s_{2}+1\right)}{n+s_{1}+1}+D_{(n, m)}^{q} B\left(n+1, s_{1}+1\right) B\left(m+1, s_{2}+1\right)\right. \\
& \left.+\frac{F_{(n, m)}^{q}}{\left(n+s_{1}+1\right)\left(m+s_{2}+1\right)}+\frac{I_{(n, m)}^{q} B\left(n+1, s_{1}+1\right)}{m+s_{2}+1}\right]^{\frac{1}{q}} \\
& +\left[\frac{G_{(n, m)}^{q} B\left(n+1, s_{1}+1\right)}{m+s_{2}+1}+C_{(n, m)}^{q} B\left(n+1, s_{1}+1\right) B\left(m+1, s_{2}+1\right)\right. \\
& \left.+\frac{J_{(n, m)}^{q} B\left(m+1, s_{2}+1\right)}{n+s_{1}+1}+\frac{F_{(n, m)}^{q}}{\left(n+s_{1}+1\right)\left(m+s_{2}+1\right)}\right]^{\frac{1}{q}} \\
& +\left[\frac{F_{(n, m)}^{q}}{\left(n+s_{1}+1\right)\left(m+s_{2}+1\right)}+\frac{I_{(n, m)}^{q} B\left(n+1, s_{1}+1\right)}{m+s_{2}+1}+\frac{J_{(n, m)}^{q} B\left(m+1, s_{2}+1\right)}{n+s_{1}+1}\right. \\
& \left.\left.+E_{(n, m)}^{q} B\left(n+1, s_{1}+1\right) B\left(m+1, s_{2}+1\right)\right]^{\frac{1}{q}}\right\},
\end{aligned}
$$

where $P(t)$ and $Q(r)$ are as defined in Theorem 2.4.

Proof. By letting $x \mapsto \frac{a+b}{2}$ and $y \mapsto \frac{c+d}{2}$ in Theorem 2.1, using the properties of the absolute value, we obtain

$$
\begin{aligned}
\mid & -\sum_{k=0}^{n-1} \sum_{l=0}^{m-1} \frac{\left[1+(-1)^{k}\right]\left[1+(-1)^{l}\right]}{2^{k+l+2}} \frac{(b-a)^{k}(d-c)^{l}}{(k+1) !(l+1) !} \frac{\partial^{k+l} f\left(\frac{a+b}{2}, \frac{c+d}{2}\right)}{\partial x^{k} \partial y^{l}} \\
& +\frac{1}{(b-a)(d-c)} \int_{a}^{b} \int_{c}^{d} f(t, r) d r d t \\
& +\frac{(-1)^{m+1}}{(d-c) m !} \sum_{k=0}^{n-1} \frac{\left[1+(-1)^{k}\right](b-a)^{k}}{2^{k+1}(k+1) !} \int_{c}^{d} Q(r) \frac{\partial^{k+m} f\left(\frac{a+b}{2}, r\right)}{\partial x^{k} \partial r^{m}} d r \\
& +\frac{(-1)^{n+1}}{(b-a) n !} \sum_{l=0}^{m-1} \frac{\left[1+(-1)^{l}\right](d-c)^{l}}{2^{l+1}(l+1) !} \int_{a}^{b} P(t) \frac{\partial^{n+l} f\left(t, \frac{c+d}{2}\right)}{\partial t^{n} \partial y^{l}} d t \mid
\end{aligned}
$$




$$
\begin{aligned}
\leq & \frac{1}{(b-a)(d-c) m ! n !}\left[\int_{a}^{\frac{a+b}{2}} \int_{c}^{\frac{c+d}{2}}(t-a)^{n}(r-c)^{m}\left|\frac{\partial^{n+m} f(t, r)}{\partial t^{n} \partial r^{m}}\right| d r d t\right. \\
& +\int_{\frac{a+b}{2}}^{b} \int_{c}^{\frac{c+d}{2}}(b-t)^{n}(r-c)^{m}\left|\frac{\partial^{n+m} f(t, r)}{\partial t^{n} \partial r^{m}}\right| d r d t \\
& +\int_{a}^{\frac{a+b}{2}} \int_{\frac{c+d}{2}}^{d}(t-a)^{n}(d-r)^{m}\left|\frac{\partial^{n+m} f(t, r)}{\partial t^{n} \partial r^{m}}\right| d r d t \\
& \left.+\int_{\frac{a+b}{2}}^{b} \int_{\frac{c+d}{2}}^{d}(b-t)^{n}(d-r)^{m}\left|\frac{\partial^{n+m} f(t, r)}{\partial t^{n} \partial r^{m}}\right| d r d t\right] .
\end{aligned}
$$

Using the power-mean inequality for each integral on the right-side of (2.29) and by the similar arguments as in proving Theorem 2.4, we get (2.28).

Corollary 2.6. If the conditions of Theorem 2.5 are satisfied and if $m=n=1$ and $s_{1}=s_{2}=1$, then we have the inequality

$$
\begin{aligned}
& \mid \frac{1}{(b-a)(d-c)} \int_{a}^{b} \int_{c}^{d} f(t, r) d r d t+\left(\frac{a+b}{2}, \frac{c+d}{2}\right) \\
& \quad-\frac{1}{2(d-c)} \int_{c}^{d} f\left(\frac{a+b}{2}, r\right) d r-\frac{1}{2(b-a)} \int_{a}^{b} f\left(t, \frac{c+d}{2}\right) d t \mid \\
& \leq\left(\frac{1}{4}\right)^{2-\frac{1}{q}}\left(\frac{b-a}{2}\right)\left(\frac{d-c}{2}\right)\left\{\left[\frac{1}{36} B_{(1,1)}^{q}+\frac{1}{18} G_{(1,1)}^{q}+\frac{1}{18} H_{(1,1)}^{q}+\frac{1}{9} F_{(1,1)}^{q}\right]^{\frac{1}{q}}\right. \\
& +\left[\frac{1}{18} H_{(1,1)}^{q}+\frac{1}{36} D_{(1,1)}^{q}+\frac{1}{9} F_{(1,1)}^{q}+\frac{1}{18} I_{(1,1)}^{q}\right]^{\frac{1}{q}} \\
& +\left[\frac{1}{18} G_{(1,1)}^{q}+\frac{1}{36} C_{(1,1)}^{q}+\frac{1}{18} J_{(1,1)}^{q}+\frac{1}{9} F_{(1,1)}^{q}\right]^{\frac{1}{q}} \\
& \left.+\left[\frac{1}{9} F_{(1,1)}^{q}+\frac{1}{18} I_{(1,1)}^{q}+\frac{1}{18} J_{(1,1)}^{q}+\frac{1}{36} E_{(1,1)}^{q}\right]^{\frac{1}{q}}\right\} .
\end{aligned}
$$

If we use the Hölder's inequality instead of the power-mean inequality we get the following result.

Theorem 2.6. Let $f: \Delta \subset[0, \infty) \times[0, \infty) \rightarrow[0, \infty), a<b, c<d$, be a continuous mapping such that $\frac{\partial^{m+n} f}{\partial t^{n} \partial r^{m}}$ exist on $\Delta^{\circ}$ and $\frac{\partial^{m+n} f}{\partial t^{n} \partial r^{m}} \in L(\Delta)$. If $\left|\frac{\partial^{n+m} f}{\partial t^{n} \partial s^{m}}\right|^{p}, p>1$, is $s$-convex on the co-ordinates on $\Delta, s_{1}, s_{2} \in(0,1]$ with $s=\frac{s_{1}+s_{2}}{2}, m, n \in \mathbb{N}, m, n \geq 1$. 
Then for $P(t)$ and $Q(r)$ defined as in Theorem 2.4 and $\frac{1}{p}+\frac{1}{q}=1$ we have

$$
\begin{aligned}
& \mid \frac{1}{(b-a)(d-c)} \int_{a}^{b} \int_{c}^{d} f(t, r) d r d t \\
& \quad-\sum_{k=0}^{n-1} \sum_{l=0}^{m-1} \frac{\left[1+(-1)^{k}\right]\left[1+(-1)^{l}\right]}{2^{k+l+2}} \frac{(b-a)^{k}(d-c)^{l}}{(k+1) !(l+1) !} \frac{\partial^{k+l} f\left(\frac{a+b}{2}, \frac{c+d}{2}\right)}{\partial x^{k} \partial y^{l}} \\
& \quad+\frac{(-1)^{m+1}}{(d-c) m !} \sum_{k=0}^{n-1} \frac{\left[1+(-1)^{k}\right](b-a)^{k}}{2^{k+1}(k+1) !} \int_{c}^{d} Q(r) \frac{\partial^{k+m} f\left(\frac{a+b}{2}, r\right)}{\partial x^{k} \partial r^{m}} d r \\
& \quad+\frac{(-1)^{n+1}}{(b-a) n !} \sum_{l=0}^{m-1} \frac{\left[1+(-1)^{l}\right](d-c)^{l}}{2^{l+1}(l+1) !} \int_{a}^{b} P(t) \frac{\partial^{n+l} f\left(t, \frac{c+d}{2}\right)}{\partial t^{n} \partial y^{l}} d t \mid \\
& \leq \frac{(b-a)^{n}(d-c)^{m}}{2^{n+m} n ! m ![(n p+1)(m p+1)]^{\frac{1}{p}}}\left[\frac{1}{2}\left(\frac{1}{\left(s_{1}+1\right)^{2}}+\frac{1}{\left(s_{2}+1\right)^{2}}\right)\right]^{\frac{1}{q}} \\
& \times\left[B_{(n, m)}^{q}+C_{(n, m)}^{q}+D_{(n, m)}^{q}+E_{(n, m)}^{q}\right]^{\frac{1}{q}} .
\end{aligned}
$$

Proof. The inequality (2.30) follows from the Hölder's inequality and (1.7).

Corollary 2.7. Under the assumptions of Theorem 2.6, if $m=n=1$ and $s_{1}=s_{2}=$ 1 , then for $\frac{1}{p}+\frac{1}{q}=1$ we have the inequality

$$
\begin{aligned}
& \mid \frac{1}{(b-a)(d-c)} \int_{a}^{b} \int_{c}^{d} f(t, r) d r d t+f\left(\frac{a+b}{2}, \frac{c+d}{2}\right) \\
& \quad-\frac{1}{2(d-c)} \int_{c}^{d} f\left(\frac{a+b}{2}, r\right) d r-\frac{1}{2(b-a)} \int_{a}^{b} f\left(t, \frac{c+d}{2}\right) d t \mid \\
& \leq \frac{(b-a)(d-c)}{2^{2+\frac{2}{q}}(p+1)^{\frac{2}{p}}} \sqrt[q]{\left|\frac{\partial^{2} f(a, c)}{\partial t \partial r}\right|^{q}+\left|\frac{\partial^{2} f(b, c)}{\partial t \partial r}\right|^{q}+\left|\frac{\partial^{2} f(a, d)}{\partial t \partial r}\right|^{q}+\left|\frac{\partial^{2} f(b, d)}{\partial t \partial r}\right|^{q}} .
\end{aligned}
$$

Our last result is for the $s$-concave functions can be stated as follows.

Theorem 2.7. Let $f: \Delta \subset[0, \infty) \times[0, \infty) \rightarrow[0, \infty), a<b, c<d$, be a continuous mapping such that $\frac{\partial^{m+n} f}{\partial t^{n} \partial r^{m}}$ exist on $\Delta^{\circ}$ and $\frac{\partial^{m+n} f}{\partial t^{n} \partial r^{m}} \in L(\Delta)$. If $\left|\frac{\partial^{n+m} f}{\partial t^{n} \partial s^{m}}\right|^{p}, p>1$, is sconcave on the co-ordinates on $\Delta, s_{1}, s_{2} \in(0,1]$ with $s=\frac{s_{1}+s_{2}}{2}, m, n \in \mathbb{N}, m, n \geq 1$. Then for $P(t)$ and $Q(r)$ defined as in Theorem 2.4 and $\frac{1}{p}+\frac{1}{q}=1$ we have 


$$
\begin{aligned}
& \mid \frac{1}{(b-a)(d-c)} \int_{a}^{b} \int_{c}^{d} f(t, r) d r d t \\
& -\sum_{k=0}^{n-1} \sum_{l=0}^{m-1} \frac{\left[1+(-1)^{k}\right]\left[1+(-1)^{l}\right]}{2^{k+l+2}} \frac{(b-a)^{k}(d-c)^{l}}{(k+1) !(l+1) !} \frac{\partial^{k+l} f\left(\frac{a+b}{2}, \frac{c+d}{2}\right)}{\partial x^{k} \partial y^{l}} \\
& +\frac{(-1)^{m+1}}{(d-c) m !} \sum_{k=0}^{n-1} \frac{\left[1+(-1)^{k}\right](b-a)^{k}}{2^{k+1}(k+1) !} \int_{c}^{d} Q(r) \frac{\partial^{k+m} f\left(\frac{a+b}{2}, r\right)}{\partial x^{k} \partial r^{m}} d r \\
& +\frac{(-1)^{n+1}}{(b-a) n !} \sum_{l=0}^{m-1} \frac{\left[1+(-1)^{l}\right](d-c)^{l}}{2^{l+1}(l+1) !} \int_{a}^{b} P(t) \frac{\partial^{n+l} f\left(t, \frac{c+d}{2}\right)}{\partial t^{n} \partial y^{l}} d t \\
& \leq \frac{(b-a)^{n}(d-c)^{m}}{2^{n+m} n ! m ![(n p+1)(m p+1)]^{\frac{1}{p}}}\left[\frac{4^{s_{1}+1}+4^{s_{2}+1}}{2}\right]^{\frac{1}{q}}\left|\frac{\partial^{n+m} f\left(\frac{a+b}{2}, \frac{c+d}{2}\right)}{\partial t^{n} \partial r^{m}}\right| .
\end{aligned}
$$

Proof. The inequality (2.31) follows from the Hölder's inequality and the inequality (1.7) with inequalities in reversed direction.

Corollary 2.8. If the conditions of Theorem 2.7 are satisfied and if $m=n=1$ and $s_{1}=s_{2}=1$, then for $\frac{1}{p}+\frac{1}{q}=1$ we have the inequality

$$
\begin{aligned}
& \mid \frac{1}{(b-a)(d-c)} \int_{a}^{b} \int_{c}^{d} f(t, r) d r d t+f\left(\frac{a+b}{2}, \frac{c+d}{2}\right) \\
& \quad-\frac{1}{2(d-c)} \int_{c}^{d} f\left(\frac{a+b}{2}, r\right) d r-\frac{1}{2(b-a)} \int_{a}^{b} f\left(t, \frac{c+d}{2}\right) d t \mid \\
& \leq \frac{(b-a)(d-c)}{2^{2-\frac{4}{q}}(p+1)^{\frac{2}{p}}}\left|\frac{\partial^{2} f\left(\frac{a+b}{2}, \frac{c+d}{2}\right)}{\partial t \partial r}\right| .
\end{aligned}
$$

\section{REFERENCES}

[1] M. Alomari and M. Darus, Co-ordinated s-convex Function in the First Sense with Some Hadamard-type Inequalities, Int. J. Contemp. Math. Sciences, 3 (32) (2008), 1557-1567.

[2] M. Alomari, M. Darus and S. S. Dragomir, Inequalities Of Hermite-hadamard's Type for Functions Whose Derivatives Absolute Values Are Quasi-convex, Rgmia Research Report Collection, 12 (Suppl. 14) (2009).

[3] M. Alomari and M. Darus, The Hadamard's Inequality For s-convex Function of 2-variables on the Co-ordinates, Int. Journal Math. Analysis, 2(13) (2008), 629-638.

[4] M. Alomari and M. Darus, The Hadamard's Inequality For s-convex Functions, Int. Journal Math. Analysis, 2(13) (2008), 639-646.

[5] M. Alomari and M. Darus, Hadamard-type Inequalities For s-convex Functions, International Mathematical Forum, 3 (2008), No. 40, 1965-1975.

[6] M. Alomari and M. Darus, on the Hadamard's Inequality For Log-convex Functions on the Coordinates, J. Of Inequal. And Appl, Article Id 283147, (2009), 13 Pages. 
[7] M. Avci, H. Kavumaci and M. E. Özdemir, New Inequalities of Hermite-hadamard Type via s-convex Functions In The Second Sense with Applications, Applied Mathematics and Computation, 217 (2011), 5171-5176.

[8] S. S. Dragomir and R. P. Agarwal, Two Inequalities For Differentiable Mappings and Applications to Special Means of Real Numbers And To Trapezoidal Formula, Appl. Math. Lett. 11(5) (1998), 91-95.

[9] S. S. Dragomir, and S. Fitzpatrick, the Hadamard's Inequality for s-convex Functions in the Second Sense, Demonstratio Math., 32 (4) (1999), 687-696.

[10] S. S. Dragomir, on Hadamard's Inequality for Convex Functions on the Co-ordinates in a Rectangle from the Plane, Taiwanese Journal of Mathematics, 4 (2001), 775-788.

[11] S. S. Dragomir and C. E. M. Pearce, Selected Topics On Hermite-hadamard Inequalities and Applications, Rgmia Monographs, Victoria University, 2000. Online: [Http://www.Staff.Vu.Edu.Au/rgmia/monographs/hermite_hadamard.Html] .

[12] W.-D. Jiang, D.-E. Niu, Y. Hua and F. Qi, Generalizations of Hermite-Hadamard Inequality to n-time Differentiable Functions Which Are s-convex in the Second Sense, Analysis (Munich) 32 (2012), 1001-1012; Available Online at http://dx.doi.org/10.1524/anly.2012.1161.

[13] D. Y. Hwang, K. L. Tseng and G. S. Yang, Some Hadamard's Inequalities for Co-ordinated Convex Functions in a Rectangle from the Plane, Taiwanese Journal of Mathematics, 11 (2007), $63-73$.

[14] D. Y. Hwang, Some Inequalities for n-times Differentiable Mappings and Applications, Kyungpook, Math. J. 43 (2003), 335-343.

[15] H. Hudzik and L. Maligranda, Some Remarks on s-convex Functions, Aequationes Math., 48 (1994), 100-111.

[16] S.-hong, B.-y. Xi and F. Qi, Some New Inequalities Of Hermite-Hadamard Type for n-times Differentiable Functions Which Are m-convex, Analysis (Munich) 32 (2012), No. 3, 247-262; Available Online at Http://dx.doi.org/10.1524/anly.2012.1167.

[17] G. Hanna, Cubature Rule from a Generalized Taylor Perspective, Phd Thesis.

[18] G. Hanna, S. S. Dragomir and P. Cerone, a General Ostrowski Type Inequality for Double Integrals, Tamkang J. Math. Volume 33, Issue 4, 2002.

[19] U. S. Kirmaci, Inequalities for Differentiable Mappings And Applications to Special Means of Real Numbers to Midpoint Formula, Appl. Math. Comput. 147 (2004), 137-146.

[20] U. S. Kirmaci, M. K. Bakula, M. E. Özdemir and J. PečArić, Hadamard-type Inequalities for s-convex Functions, Appl. Math. And Compt., 93 (2007), 26-35.

[21] M. A. Latif and M. Alomari, Hadamard-type Inequalities For Product Two Convex Functions on the Co-ordinetes, Int. Math. Forum, 4(47) (2009), 2327-2338.

[22] M. A. Latifand and M. Alomari On the Hadamard-type Inequalities for $h$-convex Functions on the Co-ordinetes, Int. J. Math. Analysis, 3 (33) (2009), 1645-1656.

[23] M. A. Latif and S. S. Dragomir, on Some New Inequalities For Differentiable Co-ordinated Convex Functions, Journal of Inequalities And Applications 2012, 2012:28 Doi:10.1186/1029242x-2012-28.

[24] M. A. Latif and S. Hussain, Some New Hermite-hadamard Type Inequalities for Functions Whose Higher Order Partial Derivatives Are Co-ordinated Convex. Facta Universitatis (Niš) Ser. Math. Inform. 27(3) (2012), 321-336.

[25] M. E. Özdemir, E. Set and M. Z. Sarikaya, New Some Hadamard's Type Inequalities for Coordinated $m$-convex and $(\alpha, m)$-convex Functions, Rgmia, Res. Rep. Coll. 13 (2010), Supplement, Article 4.

[26] M. E. Özdemir, H. Kavurmaci, A. O. Akdemir and M. Avci, Inequalities for Convex and $s$-convex Functions On $\delta=[a, b] \times[c, d]$, Journal Of Inequalities and Applications 2012:20, doi:10.1186/1029-242x-2012-20. 
[27] M. E. Özdemir, M. A. Latif and A. O. Akdemir, On Some Hadamard-type Inequalities for Product of Two s-convex Functions on the Co-ordinates, Journal of Inequalities And Applications, 2012:21, doi:10.1186/1029-242x-2012-21.

[28] M. E. Özdemir, A. O. Akdemir and M. Tunc, on the Hadamard-type Inequalities for Coordinated Convex Functions, Arxiv:1203.4327v1.

[29] C. M. E. Pearce and J. E. Pečarić, Inequalities For Differentiable Mappings with Applications to Special Means and Quadrature Formula, Appl. Math. Lett. 13 (2000), 51-55.

[30] J. E. Pečarić, F. Proschan and Y. L. Tong, Convex Functions, Partial Ordering and Statistical Applications, Academic Press, New York, 1991.

[31] M. Z. Sarikaya, E. Set, M. E. Özdemir and S. S. Dragomir, New Some Hadamard's Type Inequalities for Co-ordinated Convex Functions, Arxiv:1005.0700v1 [Math.Ca].

[32] B.-Y Xi and F. Qi, Some Hermite-Hadamard Type Inequalities For Differentiable Convex Functions and Applications, Hacet. J. Math. Stat., 42: in Press, 2013.

[33] B.-Y Xi and F. Qi, Some Hermite-Hadamard Type Inequalities For Differentiable Convex Functions with Applications to Means, J. Funct. Spaces Appl., 2012: in Press, 2012; Available Online at http://dx.doi.org/10.1155/2012/980438.

School of Computational and Applied Mathematics, UNIVERSITY OF THE WITWATERSRAND,

Private Bag 3, Wits 2050, Johannesburg,

SOUTH AFricA

E-mail address: m_amer_latif@hotmail.com 